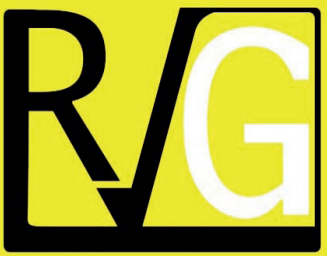

Abril-Junio, 2021

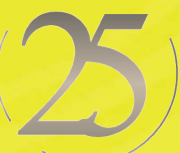

ANVERSARD
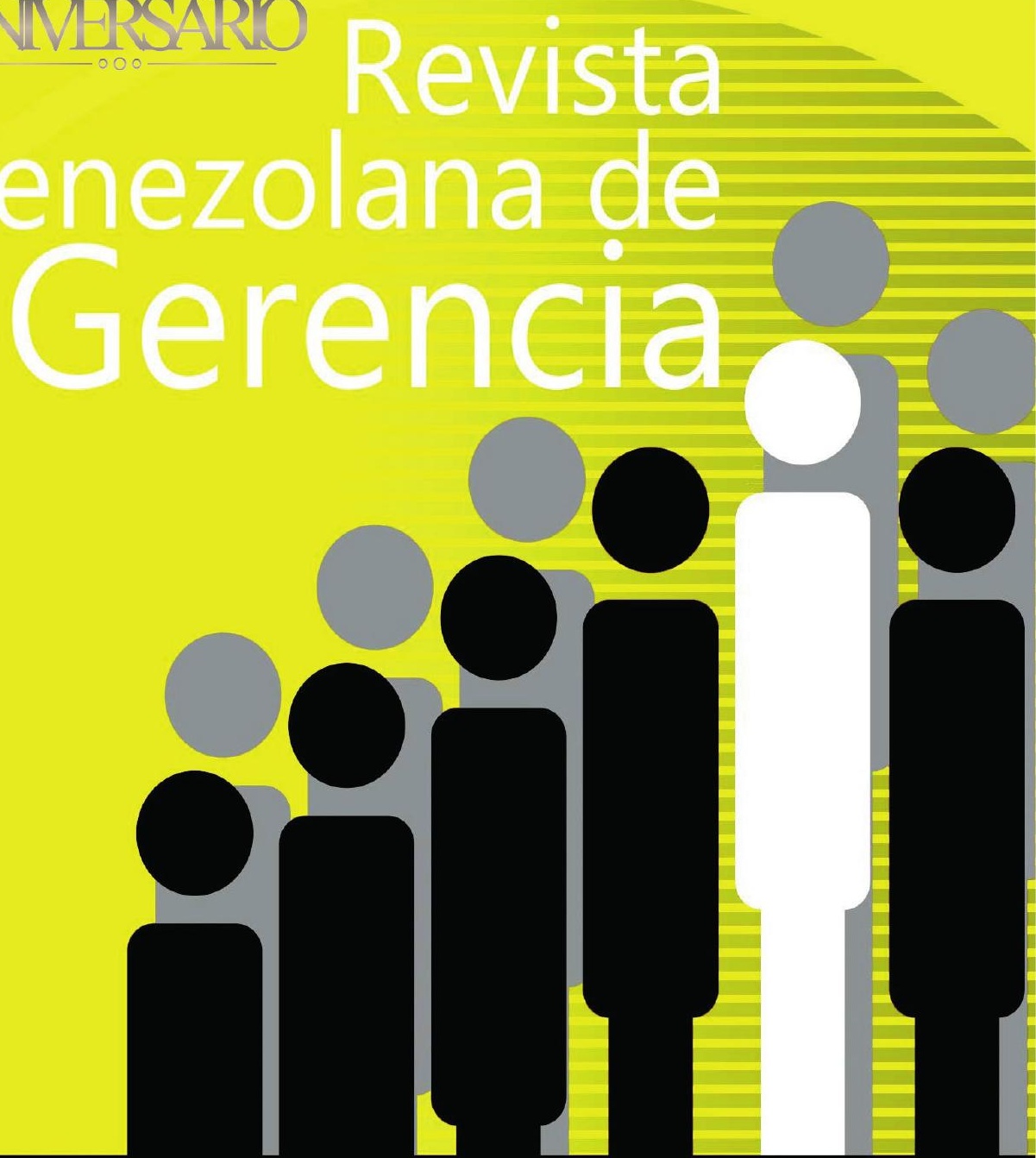


\title{
Depresión, Ansiedad, estrés en estudiantes y docentes: Análisis a partir del Covid 19
}

\author{
Cabezas-Heredia, Edmundo* \\ Herrera-Chávez, Renato* \\ Ricaurte-Ortiz, Paúl ${ }^{\star \star}$ \\ Novillo Yahuarshungo, Carlos ${ }^{* * \star}$
}

\section{Resumen}

En Febrero 2020, el virus SARS-CoV-2 procedente de China ha llegado a Ecuador, el 16 de marzo se declara el estado de excepción, llevando al confinamiento a toda la población. La presente investigación se contextualiza en estudiantes, docentes de Posgrado. El objetivo es analizar, a partir del Covid 19, los niveles de depresión, ansiedad y estrés en estudiantes y docentes de Posgrado, así como la capacidad de afrontamiento realizando un análisis en función de las variables sociodemográficas. La metodología consiste en recolectar una muestra de 139 estudiantes y docentes de la maestría en prevención de Riesgos Laborales, aplicar la encuesta DASS 21 para medir las escalas de ansiedad, estrés y depresión. El diseño experimental es de tipo transversal, correlacional e inductivo. Los resultados demuestran que el Síndrome de Trastorno Mental en sus tres escalas es leve a moderado existen casos severos con afrontamientos bajos de las personas ante la presencia del Covid 19. Referente a las variables sociodemográficas el afrontamiento es bajo ante la presencia de la crisis. Se pronostica que la sintomatología determinada aumentará según vaya transcurriendo el confinamiento y aumento de casos- muertes por Covid 19. Se defienden intervenciones mediante programas prevención psicológica. Se determina que a menor capacidad de afrontamiento mayor nivel de estrés, ansiedad y depresión estudiantes y docentes con niveles de poco a medio.

Palabras clave: Estrés; Ansiedad; Depresión; Covid 19.

Recibido: 12.07.20 Aceptado: 15.01.21

* Carrera de Agroindustria, Facultad de Ingeniería, Universidad Nacional de Chimborazo, Código Postal 060150, Riobamba - Ecuador, E-mail: ecabezas@unach.edu.ec, ORCID: https://orcid.org/0000-0001-5708$\underline{0054}$

** Carrera de Turismo, Facultad de Ciencias Políticas y Administrativas, Universidad Nacional de Chimborazo, Código Postal 060150, Riobamba - Ecuador, E-mail: rherrera@unach.edu.ec ORCID: https://orcid.org/00000002-6816-7945

*** Carrera de Agroindustria, Facultad de Ingeniería, Universidad Nacional de Chimborazo, Código Postal 060150, Riobamba - Ecuador, E-mail: pricaurte@unach.edu.ec ORCID: https://orcid.org/0000-0003-46941597

**** Carrera de Ingeniería Agroindustrial, Facultad de Ciencias Agropecuarias, Universidad Estatal de Bolívar, Código Postal 020105, Guaranda - Ecuador, E-mail: cnovillo@ueb.edu.ec ORCID: https://orcid.org/0000$\underline{0001-7146-8098}$ 


\section{Depression, Anxiety, Stress in Students and Teachers: Analysis from Covid 19}

In February 2020, the SARS-CoV-2 virus from China has reached Ecuador, on March 16 the state of exception is declared, leading to the confinement of the entire population. This research is contextualized in students, postgraduate teachers. The objective is to analyze, based on Covid 19, the levels of depression, anxiety and stress in graduate students and teachers, as well as the ability to cope by performing an analysis based on sociodemographic variables. The methodology consists of collecting a sample of 139 students and teachers of the Master's Degree in Occupational Risk Prevention, applying the DASS 21 survey to measure the anxiety, stress and depression scales. The experimental design is cross-sectional, correlational and inductive. The results show that the Mental Disorder Syndrome in its three scales is mild to moderate, there are severe cases with low coping of people in the presence of Covid 19. Regarding the sociodemographic variables, coping is low in the presence of the crisis. It is predicted that the specific symptoms will increase as the confinement progresses and the increase in cases-deaths from Covid 19. Interventions are defended through psychological prevention programs. It is determined that the lower the coping capacity, the higher the level of stress, anxiety and depression, students and teachers with levels from little to medium.

Keywords: Stress; Anxiety; Depression; Covid 19

\section{Introducción}

En Diciembre de 2019, empezó un brote de una nueva neumonía por coronavirus en Wuhan (Hubei, China) (Chen, 2020), el nuevo coronavirus (COVID-19) comenzó a extenderse por toda China. El rápido aumento de casos y muertes ha creado molestias como estrés, ansiedad y depresión, en el personal médico y población en general (Liu, 2020), provocando muerte por todo el mundo, América Latina y Ecuador.

En América Latina y el Caribe según datos de la Comisión Económica para América latina y el Caribe (CEPAL), la pandemia enfrenta una posición más débil que la del resto del mundo. Antes de la pandemia, se preveía que la región crecería un máximo del 1,3\%. (CEPAL, 2020). Los efectos de la crisis han llevado a cambiar y pronosticar una caída del PIB de al menos un $1,8 \%$. La presencia del Covid-19 afectará el número de empleos (aumento del desempleo y el subempleo), y por ende la calidad de vida (reducción de salarios y menor acceso a la seguridad social) y sobre todo inseguridad ciudadana (OIT, 2020), se estima un incremento del desempleo mundial de entre 5,3 millones de personas y 24,7 millones de personas, con una base de 188 millones de personas desocupadas en 2019.

Un escenario lúgubre para el Ecuador, América Latina y el mundo, esta pérdida de ingresos laborales se traducirá en un menor consumo de bienes y servicios, llevando a los sectores de la sociedad a situaciones de extrema pobreza. La crisis generará un mayor impacto en los sectores más vulnerables: 
en aspectos de salud, desempleo a todo nivel, colapso de los sistemas de salud, aumento de migración e inseguridad en la población.

En Ecuador el 19 de febrero de 2021, con 71.276 son casos confirmados con pruebas PCR y 10.738 personas fallecidas (confirmados COVID-19). (MSP, 2021). Los riesgos médicos, el impacto psicosocial de la pandemia es notoria. Varias líneas de investigación han trabajado en la comprensión sobre el origen, impacto de las epidemias y de cómo las afrontan, sobre todo el aspecto emocional (Idoiaga et al, 2017).

Es difícil predecir con exactitud y estimar las consecuencias psicológicas por efectos del COVID-19. Investigaciones que llegan de China, donde se originó, registran que el miedo a lo inexplorado y desconocido pueden llevar a desarrollar enfermedades mentales como: los trastornos de estrés, ansiedad, depresión, somatización y conductas que degeneran en aumento de consumo de alcohol, tabaco y otras substancias nocivas para la salud (Shigemura et el, 2020).

Del 31 de enero al 2 de febrero de 2020 se realizó un estudio con 1.210 personas en 194 ciudades de China en el que se aplicó la escala de Depresión, Ansiedad y Estrés (DASS21), para entender las variables, el nivel de impacto psicológico; ansiedad, depresión y estrés en la etapa inicial del brote de COVID-19; se encontró que el $16,5 \%$ de los encuestados mostró síntomas depresivos de moderados a graves; 28,8\% síntomas de ansiedad de moderados a graves; y $8,1 \%$ con niveles de estrés de moderados a graves (Wang et al, 2020), el mismo que se puede deber a algo nuevo y desconocido que ha generado consecuencias muy graves en la población.
Los efectos de salud por el Covid 19 se asociaron significativamente con un mayor impacto psicológico en los niveles más altos de estrés, ansiedad y depresión (Wang et al, 2020). Otra investigación en 1.354 adultos canadienses, a principios de febrero de 2020, indicó que más del $60 \%$ de encuestados estaban preocupadas por el virus y el 7\% "muy preocupadas" por la infección (Asmundson y Taylor, 2020), en ese instante de tiempo sólo había 4 canadienses infectados, es decir riesgo muy bajo; sin embargo, el $7 \%$ de la población, es decir, 2,6 millones de personas, estaba muy preocupada. A nivel mundial el miedo al COVID-19 es superior a los brotes estacionales de la gripe, aunque esta última ha matado a un número considerablemente mayor de personas (Asmundson y Taylor, 2020).

El confinamiento y el cambio de la modalidad a teletrabajo, así como la intensidad y sobre carga de trabajo, la responsabilidad del rol que desempeña, conllevaría a consecuencias de tipo emocional, cognitivas y conductuales que se traducen en estrés y se asocian a los factores psicosociales de la nueva modalidad de trabajo (Díaz, 2017).

Al realizar un análisis de la problemática en el mundo y especialmente en Ecuador resulta preocupante la presencia del Covid 19, esto ha traído enfermedad, muerte, afección en el ámbito laboral afectando la calidad de vida de la población con incremento de índices de pobreza y sobre todo la afección psicosocial por efectos de aislamiento, miedo, teletrabajo, carga laboral alta: esto hace que este tema tenga una relevancia por ser estudiada.

La presencia del Covid 19, más allá de los riesgos médicos, impacto psicológico y social de la pandemia es 
Cabezas-Heredia, Edmundo; Herrera-Chávez, Renato; Ricaurte-Ortiz, Paúl; Novillo Yahuarshungo, Carlos

Depresión, Ansiedad, estrés en estudiantes y docentes: Análisis a partir del Covid 19

incuestionable que la presencia de esta ha generado un golpe que requiere ser afrontando en todos los ámbitos sobre todo en el emocional parámetro clave para este proceso.

La emergencia de salud pública mundial requiere investigar el impacto psicológico que está produciendo la pandemia en poblaciones concretas, para desarrollar estrategias que permitan reducir el impacto psicológico en población vulnerable con sintomatología presentes en la crisis (Wang et al, 2020). El presente estudio permite medir los niveles de estrés, ansiedad y depresión en una muestra de estudiantes y docentes de Posgrado en el inicio de la pandemia y así predecir las necesidades psicológicas de la población.

Los niveles de confinamiento han originado que exista niveles mucho más altos en el estrés ansiedad y depresión (Brooks et al, 2020). El confinamiento acarrea problemas psicológicos como estrés, ansiedad y depresión (Cava et al, 2005). Se espera que las personas con enfermedades crónicas presenten niveles más altos de síntomas psicológicos (Applegate y Ouslander, 2020), el COVID-19 se presenta con mayor fuerza en personas con múltiples enfermedades subyacentes (Dong et al, 2020). Las personas adulto mayores se pronostica sean psicológicamente más vulnerables que los jóvenes en esta crisis (Idoiaga et al, 2017).

\section{Depresión, ansiedad y estrés: algunas reflexiones teóricas}

Según plantea Camargo (2020), las investigaciones en torno a la salud mental han tenido un aumento considerable en los últimos años, donde los factores psicosociales se han convertido en determinantes de supervivencia, siendo los estados emocionales positivos aspectos influyentes para los resultados de salud física (Apaza et al, 2020), la presencia del Covid 19 hace que estos aspectos psicológicos cambien en desmedro del estado físico de la persona que requiere atención médica y psicológica en ciertos casos.

La depresión como patología se presenta para atención clínica y psicológica que puede convertirse en un problema serio de salud mental que puede afrontarse hasta llevar al suicidio. Se considera la depresión, como trastorno mental y emocional ( $\mathrm{Li}$, Theng \& Foo, 2015). Surge de una interacción de factores biológicos, psicológicos y sociales (Ellis et al, 2017); en que los factores de riesgo se típica a adultos mayores (65 años y más), asumen la soledad como una condición psicosocial permanente en ellos por el descuido familiar y falta de cuidado - cariño.

La ansiedad y la depresión se consideran como desórdenes altos en centros de salud y en la población en general, los centros universitarios en los Departamentos de Bienestar estudiantil son los más atendidos. Pardo etal, (2004), la American Psychological Association (APA, 2018), establece que la causa de la depresión se debe a cambios químicos que están presentes en el organismo influyendo de manera negativa en las emociones y en los procesos del pensamiento, para muchas personas, la depresión muestra primariamente la ausencia de equilibrio en determinados aspectos mentales y emocionales de la vida. Las personas con depresión se sienten desamparadas, abrumadas sin esperanza y se culpan por tener estos sentimientos negativos, algunas tienen 
pensamientos de suicidio, esto se ve agravado con la presencia del Covid 19 en el mundo generando sintomatología clínica en los individuos afectados por la pandemia.

La ansiedad, proviene de la apreciación de eventos no controlables, ante los cuales se sugiere realizar actividades de resilencia y estilos de vida saludables como cuidar la higiene, comer saludable, hacer ejercicios, bailar, leer libros, entre otros (Mukhtar, 2020). El confinamiento provoca mucha ansiedad en la población de diferentes países (Thakur y Jain, 2020), en la que establece que la población más vulnerable son los que tienen problemas de salud mental (depresión), y adultos mayores que viven en soledad $y$ aislamiento; con características de afrontamiento suicida.

La ansiedad es parte de la existencia humana, todas las personas tiene un grado moderado con una respuesta de afrontamiento muchas veces positivo y otras negativas (Sierra, Ortegall y 2003). Según la American Psychological Association (APA, 2018), se establece que en algún momento sentimos como una emoción negativa, es decir, es una reacción humana normal que se da ante situaciones estresantes como este caso la presencia del Covid 19. La ansiedad leve mantiene en un estado de alerta y concentración. Las personas con trastorno de ansiedad, sienten demasiado temor y preocupación, lo cual debilita y no deja realizar las actividades diarias de las personas. En efecto la ansiedad genera graves problemas, como dificultades en el trabajo con respecto al desempeño laboral además de tener relaciones tirantes con la familia, amigos y compañeros y ahora con el confinamiento y volver a una realidad diferente por presencia del Covid 19.
El estrés afecta a las personas en el transcurso de la vida ante diferentes circunstancias de la misma. Los estudiantes en edad joven, el estrés se considera una problemática importante a nivel mundial (Bedoyalau, Matos y Zelaya, 2014), se refleja reacciones psicológicas, físicas y de comportamiento ante las situaciones propias de la actividad diaria. Al respecto, Apaza et al, (2020) señalan que el estrés se divide en etapas: elementos ambientales, familiares, aspectos laborales e interpersonales que al combinarse con la pandemia tener diferentes características de presentación agravadas con el confinamiento en diferentes patologías o sintomatologías.

A nivel estudiantil se puede presentar en forma de somnolencia, inquietud y aumento o reducción del consumo de alimentos u otro tipo de substancias que pueden ser nocivas para la salud (Hugo et al, 2014). Desde la posición de Beery y Kaufer (2015), convivimos con el estrés por la presencia de diversidad de agentes estresores cambiando las condiciones de vida $y$ afectando al entorno social, el virus ha afectado en todo ámbito: psicológico, aislamiento, pobreza, desempleo Según American Psychological Association (APA, 2018), se describe que los síntomas del estrés son agobio, agotamiento continuo y preocupación persistente. El estrés puede afectar a cualquier persona, sin importar género, edad ni sexo, puede llegar a perjudicar en la salud: física como mental y disminuir las defensas de su sistema inmunológico, siendo más propenso a tener una enfermedad. E estrés es molestosa produce cambios bioquímicos, fisiológicos y conductuales.

El estrés tiene gran impacto, $y$ definen el término de estrés como un 
Cabezas-Heredia, Edmundo; Herrera-Chávez, Renato; Ricaurte-Ortiz, Paúl; Novillo Yahuarshungo, Carlos

Depresión, Ansiedad, estrés en estudiantes y docentes: Análisis a partir del Covid 19

estado de mecanismos fisiológicos del que tiene el organismo para mantener un equilibrio, es decir, homeostasis (Román y Hernández, 2011). Para Regueiro et al, (2015) la respuesta al estrés es la forma particular de afrontar y adaptarse a varias situaciones y demandas que nos vamos encontrando, actuando como respuesta la cual aparece cuando pensamos que nuestros recursos son insuficientes para solucionar el problema.

Para Castellanos, (2020), el COVID 19 ha generado efectos negativos a nivel mundial, ha teniendo impactos profundos en la morbilidad, en pérdidas humanas y en el comportamiento social; en lo económico ha producido simultáneamente un shock de oferta (cese de la actividad económica) con un shock de demanda (caída de los ingresos y por ende del consumo), afectando profundamente la economía mundial como no se ha visto en la últimas décadas(de hecho la coincidencia de shock de oferta y demanda no son frecuentes en las economías), esto agudiza la crisis familiar de su entorno en trastornos de índole psicológico presentes en estrés, ansiedad y depresión que necesita de resilencia para salir de la crisis.

Para Apaza et al, (2020) las medidas inadecuadas para enfrentar al Covid 19 pueden conducir a trastornos psicológicos como el estrés postraumático, depresión, ansiedad, trastornos de pánico y de conducta, por factores como separación de la familia, dolor, duelo, soledad, entre otros siendo la problemática a solucionar. Aunque el mundo ya se ha enfrentado a extensas epidemias de enfermedades respiratorias agudas como el SARS en 2003, este brote se manejó adecuadamente con medidas de cuarentena. Además de la consiguiente inestabilidad social (Chen,
2020), COVID-19 influye de manera persistente en todos los aspectos de la vida humana (Makamure, et al, 2013). Personas de todo el mundo están experimentando emociones duras en la actividad diaria. Puede ocurrir una situación alarmante cuando nuestra gente puede dejar de depender de la validez de los servicios de salud mental debido a la exacerbación de la ansiedad y estrés entre ellos (Van Bortel, et al, 2016).

Por lo tanto, el presente estudio plantea como hipótesis de investigación: Si existe relación entre los niveles de depresión, ansiedad y estrés ante la capacidad de afrontamiento del Covid 19. Este estudio tiene como objetivo identificar los trastornos psicológicos de ansiedad, depresión y estrés en niveles de comportamiento con el fin de estimar la gravedad del mismo y generar bienestar psicológico de la comunidad universitaria mediante primeros auxilios psicológico y atención preventiva. Esta investigación facilitaría en gran medida a nuestros maestrantes conocer sobre el fenómeno y formular políticas adecuadas para abordar científicamente y profesionalmente formas de actuación ante el fenómeno de estudio.

\section{Aspectos metodológicos de la investigación}

El estudio es de tipo exploratoriodescriptivo de tipo trasversal. Las técnicas empleadas para recolectar la data y por la presencia del COVID-19, se aplicó un cuestionario en Google Forms que se difundió a través del link a los WhatsApp de los presidentes de curso y de docentes amigos. En total se enviaron a aproximadamente 400 personas, de las cuales respondieron 139. La base de datos en SPSS V.24 
se programó y se realizó un análisis de los ítems de respuesta por pregunta, dimensión de los sujetos encuestados. Los cuestionarios al inicio de la encuesta se informó que eran anónimos y de carácter voluntario.

DASS-21 (Ruiz, et al, 2017), fue la escala utilizada con ítems relacionados con las variables sociodemográficas de los y las participantes.

La escala DASS-21 está compuesta por 21 ítems con escala de Likert. Los factores: Depresión contiene: (ítems: 3, $5,10,13,16,17$ y 21), Ansiedad: (ítems: 2, 4, 7, 9, 15, 19 y 20) y Estrés: (ítems: 1, 6, 8, 11, 12, 14 y 18). (Antúnez Z, Vinet EV., 2012).

La categorización de las 3 dimensiones se ha obtenido mediante el sumatorio de las puntuaciones de las respuestas obtenidas en los ítems correspondientes a cada uno de los factores, para lo cual en la programación establecer los promedios y evaluar los niveles de riesgo.

Las opciones de respuesta de la escala de lickers fueron: 0 : no me ha ocurrido; 1: me ha ocurrido un poco, o durante parte del tiempo; 2: me ha ocurrido bastante, o durante una buena parte del tiempo; y 3 : me ha ocurrido mucho, o la mayor parte del tiempo. Con preguntas tipo: "Me resulto difícil calmarme", "Era consciente de la sequedad de mi boca", entre otras. Se categorizaron con los puntos de corte. $Y$ se determinó los niveles de riesgo de los síntomas depresivos, de ansiedad y de estrés y poder así analizar su sintomatología.

La fiabilidad de la escala del test Dass 21 se lo hace mediante una prueba piloto en el que el coeficiente alfa de Cronbach $(\alpha)$, de todo el test siendo $\alpha=0,987$ que garantiza la validez del cuestionario, de esta misma escala, donde se pudo comprobar la relación estrecha, positiva y grande que tenían los factores entre sí. Además de la correlación entre los factores objeto de estudio, se tuvieron presentes estudios de validez convergente y discriminante de la escala anteriormente, para garantizar su fiabilidad y validez en este estudio.

En el SPSS V24 se recolecto los datos sociodemográficos de los y las participantes, diseñado con respuestas cerradas, se preguntó sobre su edad, sexo entre otros, adicionalmente se presentó un cuestionario para medir el nivel de afrontamiento de las personas sujetas de estudio ante la Covid 19.

Con los datos obtenidos en ambos cuestionarios se realizaron análisis descriptivos, para estudiar las frecuencias de los síntomas de depresión, ansiedad y estrés estratificados por variables sociodemográficas y comparados con el nivel de afrontamiento de las personas.

\section{Niveles de depresión, ansiedad y estrés ante la presencia del Covid: caso de estudio}

La aparición del COVID-19 ha traído efectos devastadores sobre la economía mundial y nacional, esta enfermedad pone en riesgo la salud humana, afecto a la oferta y a la demanda, interrumpió las cadenas de producción, genero pérdida de ingresos y de ganancias debido a un alza del desempleo y mayores dificultades para cumplir con las deudas adquiridas por la ciudadanía, así como factores de orden psicológico a las personas.

En la totalidad de este estudio participaron 139 personas estudiantes y docentes del Programa de Maestría en 
Cabezas-Heredia, Edmundo; Herrera-Chávez, Renato; Ricaurte-Ortiz, Paúl;

Novillo Yahuarshungo, Carlos

Depresión, Ansiedad, estrés en estudiantes y docentes: Análisis a partir del Covid 19

Prevención de Riesgos laborales de la UTPL. Las muestras fueron reclutadas en Junio del 2020 en la ciudad de Loja por Regiones Sierra y Costa de los maestrantes y docentes.

La tabla 1, presenta las variables sociodemográficas de las personas consultadas en la encuesta.

\section{Tabla 1}

\section{Variables Sociodemográficas}

\begin{tabular}{ll}
\hline Variables Sociodemográficas & Frecuencia y Porcentajes \\
\hline \multirow{2}{*}{ Género: } & $71.2 \%(n=90)$ hombres \\
& $28.8 \%(n=40)$ mujeres. \\
\hline \multirow{3}{*}{ Estado Civil: } & $38.1 \%$ solteros $(n=53)$ \\
& $58.3 \%$ casados $(n=81)$ \\
& $3.6 \%$ divorciados $(n=5)$. \\
\hline \multirow{2}{*}{ Sector de Residencia: } & $88.5 \%(n=123)$ viven en el sector Urbano \\
& $11.5 \%(n=16)$ en el sector rural. \\
\hline \multirow{2}{*}{ Nivel de Educación: } & $97.1 \%(n=135$ son estudiantes $)$ \\
& $2.9 \%(n=4)$ docentes de Posgrado. \\
\hline & Hasta 22 años $0.7 \%(n=1)$ \\
Edad: & De 23 a 32 años el $49.6 \%(n=69)$ \\
& De 33 a 42 años el $39.6 \%(n=55)$ \\
& De 43 a 52 años el $7.9 \%(n=11)$ \\
& De 53 a 62 años el $2.2 \%(n=3)$ \\
\hline &
\end{tabular}

Fuente: Elaboración propia

- Depresión:

La gráfica 1 presenta los datos de depresión de los encuestados por presencia del COVID - 19 en el momento de la aplicación.

\section{Gráfica 1 \\ Niveles de Depresión de los encuestados ante el COVID - 19}

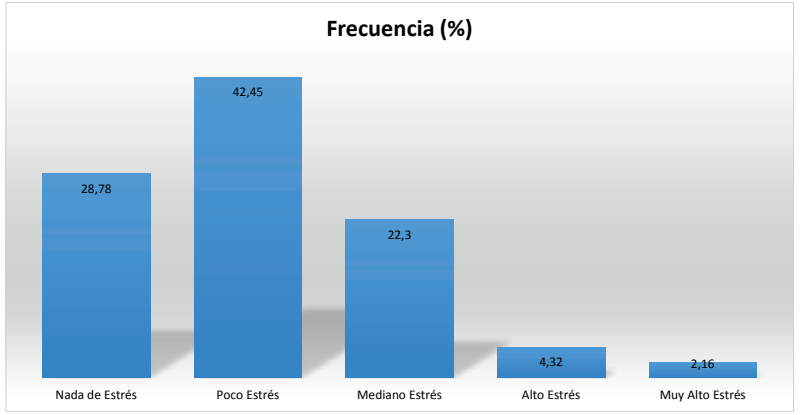

Fuente: Elaboración propia 
Los niveles de Depresión presentes en los encuestados en su mayoría en ausencia y poca depresión, sin embargo existe un \% importante entre alto y muy alto que debe ser atendido inmediatamente medicamente como psicológicamente.

\section{- Ansiedad:}

La gráfica 2 presenta los datos de Ansiedad de los encuestados por presencia del COVID - 19 en el momento de la aplicación.

\section{Gráfica 2}

Niveles de Ansiedad de los encuestados ante el COVID - 19.

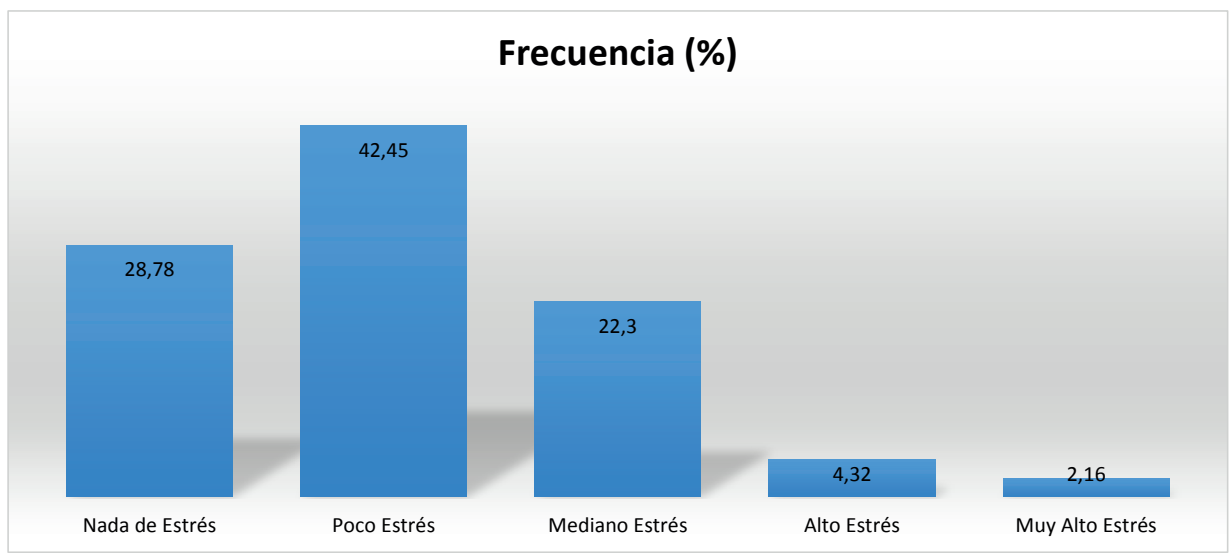

Fuente: Elaboración propia

Los niveles de Ansiedad presentes en los encuestados en su mayoría se encuentran en nada y poca ausencia y poca ansiedad en su mayoría, sin embargo existe un \% pequeño entre alto y muy alto que debe ser considerado su análisis y atención.

La gráfica 3 presenta los datos de Estrés de los encuestados por presencia del COVID - 19 en el momento de la aplicación. 
Cabezas-Heredia, Edmundo; Herrera-Chávez, Renato; Ricaurte-Ortiz, Paúl;

Novillo Yahuarshungo, Carlos

Depresión, Ansiedad, estrés en estudiantes y docentes: Análisis a partir del Covid 19

- Estrés:

\section{Gráfica 3 \\ Niveles de Estrés de los encuestados ante el COVID - 19}

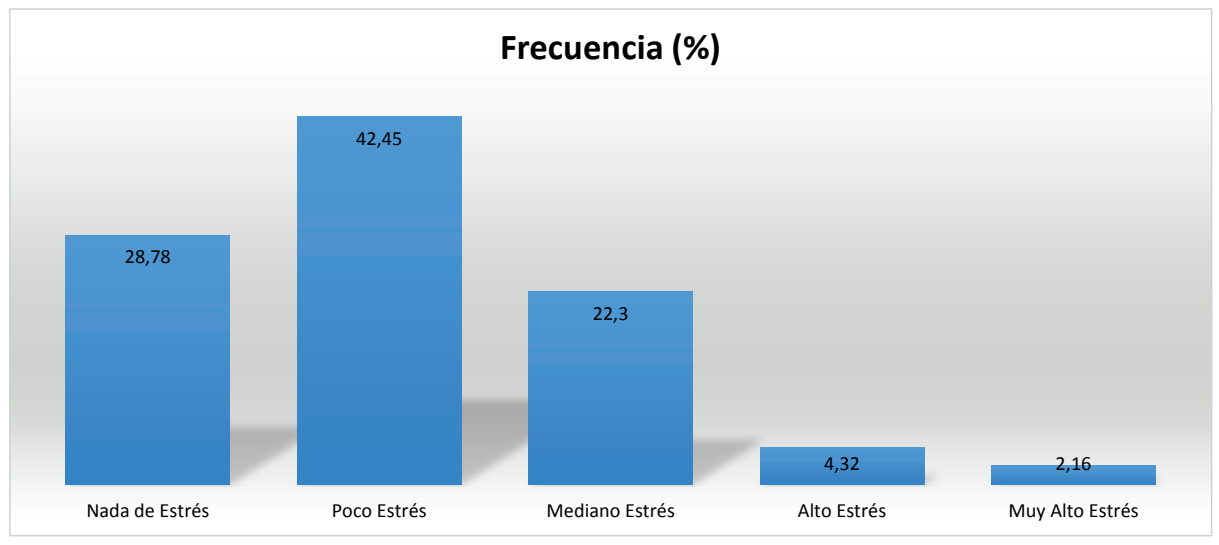

Fuente: Elaboración propia

Los niveles de Estrés presentes en los encuestados en su mayoría en nada y poca ausencia y poca ansiedad en su mayoría, sin embargo existe un \% pequeño entre alto y muy alto que debe ser considerado su análisis y prevención.

Los Porcentajes de niveles severos y extremadamente severos de estrés, ansiedad y depresión en la muestra recolectada en estudiantes y docentes de Postgrado de la Universidad Técnica Particular de Loja fueron menores que los recogidos en el estudio realizado en China (Wang et al, 2020). Este dato requiere ser analizado, teniendo en cuenta que el cuestionario se realizó en fase inicial del brote de COVID-19 en el Ecuador.

Esto podría generar interrogantes y explicaciones en vista de que los estudiantes y docentes de Postgrado de la Maestría en Prevención de Riesgos laborales de la UTPL, tenían conocimiento sobre el virus, por su tiempo de llegada al país posterior que a China este conocimiento de la pandemia podría explicar que los niveles de estrés, ansiedad y depresión fueran menores.

Además, la recolección de la muestra en los primeros meses de la pandemia en el país, los casos eran escasos, y la confianza de que llegue a todo los sectores del país generaba duda y exceso de confianza, ya que todavía la epidemia a un problema lejano que afecta a otros países como la China (Joffe, 2011).

\section{- Síndrome de Trastorno Mental (Dass 21):}

La tabla 2, presenta los niveles del Síndrome de Trastorno Mental con el test DASS 21, de las personas consultadas en la encuesta. 
Tabla 2

Niveles del Síndrome de Trastorno Mental (Dass 21)

\begin{tabular}{lcc}
\hline Niveles DASS 21 & Frecuencia & Porcentaje (\%) \\
\hline Leve Síndrome de Trastorno Mental & 75 & 54,0 \\
\hline Moderado Síndrome de Trastorno Mental & 36 & 25,9 \\
\hline Severo Síndrome de Trastorno Mental & 6 & 4,3 \\
\hline Muy Severo Síndrome de Trastorno Mental & 4 & 2,9 \\
\hline Total & 139 & 100,0 \\
\hline
\end{tabular}

Fuente: Elaboración propia

La muestra analizada existen medias superiores de niveles de estrés, ansiedad y depresión después de experimentar el tiempo de cuarentena y presencia del Covid 19. Por lo que, a la población conforme avance el tiempo para conocer, aprender y convivir el proceso de crisis a la que se están enfrentando y la vacunación estos disminuirán. Además, es evidente que estos niveles irán en aumento según se vaya alargando el tiempo de confinamiento y aislamiento, por lo que sería importante continuar la evaluación para observar el desarrollo del fenómeno de estudio (Brooks et al, 2020).

En los resultados de este estudio, se han encontrado medias superiores entre leve y moderado en estrés, ansiedad y depresión entre 23 a 32 años, seguidos de 33 a $42 \mathrm{y}$, finalmente, la media en las tres dimensiones es inferior de los 43 en adelante. Los estudiantes de postgrado medianamente joven tienen la necesidad de adaptarse al nuevo contexto educativo sin clases presenciales complicando el proceso de enseñanza aprendizaje (Antúnez y
Vinet, 2012).

En este sentido, las instituciones educativas pusieron en marcha estrategias educativas online desde un primer momento y la UTPL no es la excepción, se pudo actuar de manera oportuna ante la incertidumbre de suspender los programas de maestría. Por ello, la población en general ante la presencia del virus es vulnerable, tiende a desarrollar trastornos emocionales, siendo necesario la prevención y los programas de intervención para disminuir los niveles de estrés creados, promovidos desde las mismas instituciones educativas serán fundamentales (Cova et al, 2007).

Los niveles del Síndrome de Trastorno Mental basados en el test Dass 21 presentes en los encuestados en su mayoría es leve, sin embargo un $\%$ significativo se encuentra entre moderado a muy severo que requiere de atención inmediata. La tabla 3, presenta la capacidad de los estudiantes y docentes para afrontar la presencia del Covid - 19 . 
Cabezas-Heredia, Edmundo; Herrera-Chávez, Renato; Ricaurte-Ortiz, Paúl;

Novillo Yahuarshungo, Carlos

Depresión, Ansiedad, estrés en estudiantes y docentes: Análisis a partir del Covid 19

Tabla 3

Capacidad de afrontamiento de estudiantes y docentes ante el Covid 19

\begin{tabular}{lcc}
\hline Denominación & Frecuencia & Porcentaje (\%) \\
\hline Afrontamiento alto & 3 & 2.2 \\
\hline Afrontamiento Mediano & 30 & 21.6 \\
\hline Poco afrontamiento & 62 & 44.6 \\
\hline Ningún Afrontamiento & 44 & 31.7 \\
\hline Total & 139 & 100.0 \\
\hline
\end{tabular}

Fuente: Elaboración propia

La capacidad para afrontar la Covid 19 desde el punto de vista de estudiantes y docentes presenta una poca capacidad de afrontamiento o ningún afrontamiento, se puede deber al temor al virus y el desconocimiento al mismo. Esto complementa con datos a 23 de Febrero de 2021 (MSP, 2021), en el que existe 274673 casos confirmados y 10796 personas fallecidas y el aumento es exponencial.
- Capacidad de Afrontamiento y variables sociodemográficas:

\section{Género:}

El gráfico 4, presenta la capacidad de los estudiantes y docentes para afrontar la presencia del Covid - 19 relacionado con el género.

\section{Gráfico 4}

\section{Niveles de Afrontamiento del COVID - 19 y Género}

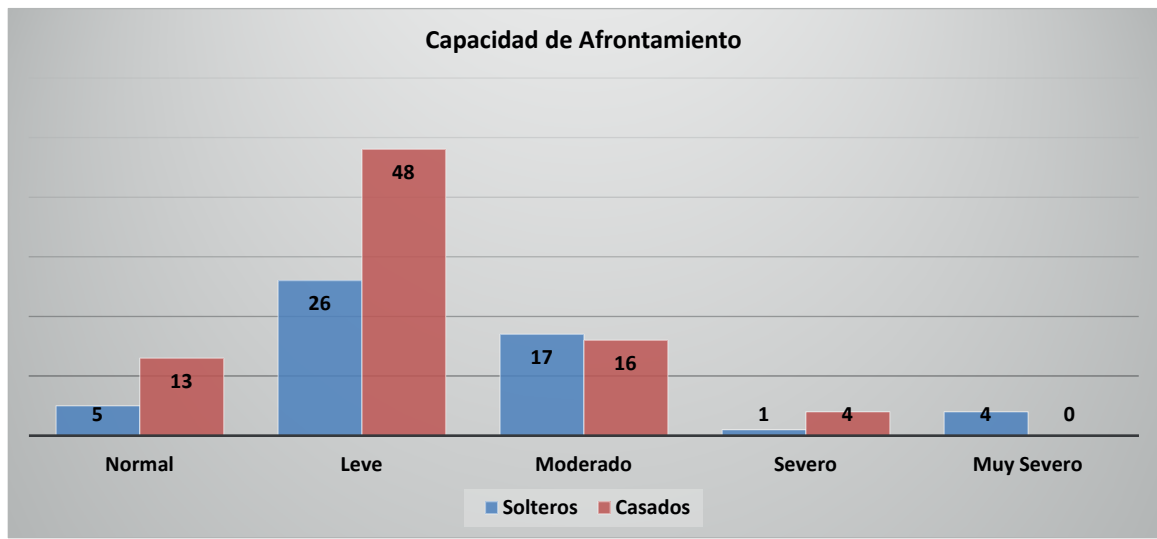

Fuente: Elaboración propia 
En la Gráfica 5 se presenta la capacidad de afrontamiento de los estudiantes y docentes relacionando con el género, se presenta que en hombres tienen una contenido mejor que la mujer para afrontar, esto implica que los efectos, sintomatología psicológica y el efecto postraumático por presencia del Covid 19 es mejor asimilado en el hombre.

El gráfico 5, presenta la capacidad de los estudiantes y docentes para afrontar la presencia del Covid - 19 relacionado con el nivel de educación.

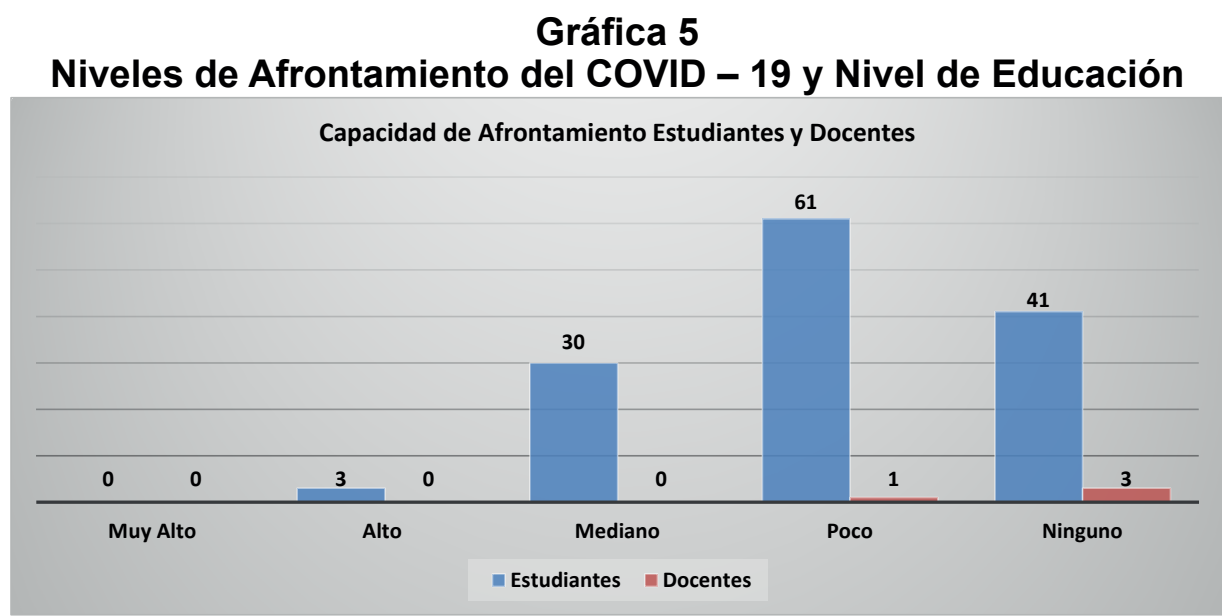

Fuente: Elaboración propia

En la Gráfica 5 se presenta la capacidad para afrontar de los estudiantes y docentes ante la presencia del Covid 19 en la que se presenta que esta es poca o ninguna en su mayoría generando que el estudiante tiene características de exceso de confianza por la presencia del virus teniendo comportamiento psicológicos diferentes a personas de mayor edad y nivel de educación.

La tabla 4, presenta la capacidad de los estudiantes y docentes para afrontar la presencia del Covid - 19 relacionado con la edad. 
Cabezas-Heredia, Edmundo; Herrera-Chávez, Renato; Ricaurte-Ortiz, Paúl; Novillo Yahuarshungo, Carlos

Depresión, Ansiedad, estrés en estudiantes y docentes: Análisis a partir del Covid 19

\section{Tabla 4}

\section{Capacidad de afrontamiento de estudiantes y docentes ante el Covid 19 por edad}

\begin{tabular}{cl}
\hline Rango de Edad (años) & Capacidad de Afrontamiento \\
\hline 13 a 22 & Alto $n=1$ \\
\hline 23 a 32 & Mediano $n=17$, Poco $n=32$, Ninguno $n=20$ \\
\hline 33 a 42 & Alto $n=1$, Mediano $n=10$, Poco $n=25$, Ninguno $n=19$ \\
\hline 43 a 52 & Mediano $n=3$, Poco $n=4$, Ninguno $n=4$ \\
\hline 53 a 62 & Alto $n=2$, Poco $n=1$ \\
\hline
\end{tabular}

Fuente: Elaboración propia.

La tabla 4 presenta la capacidad de afrontamiento de estudiantes y docentes por edad ante el Covid 19, donde se puede inferenciar que estos son de medio a bajo lo que puede generar presencia de patologías y sintomatología altas por exceso de confianza, no acatar las disposiciones de confinamiento y protección por la pandemia emita por las entidades de control.

El gráfico 6, presenta los niveles del Síndrome de Trastorno Mental (Dass 21) y el género.

\section{Gráfica 6 \\ Niveles de Síndrome de Trastorno Mental y Género}

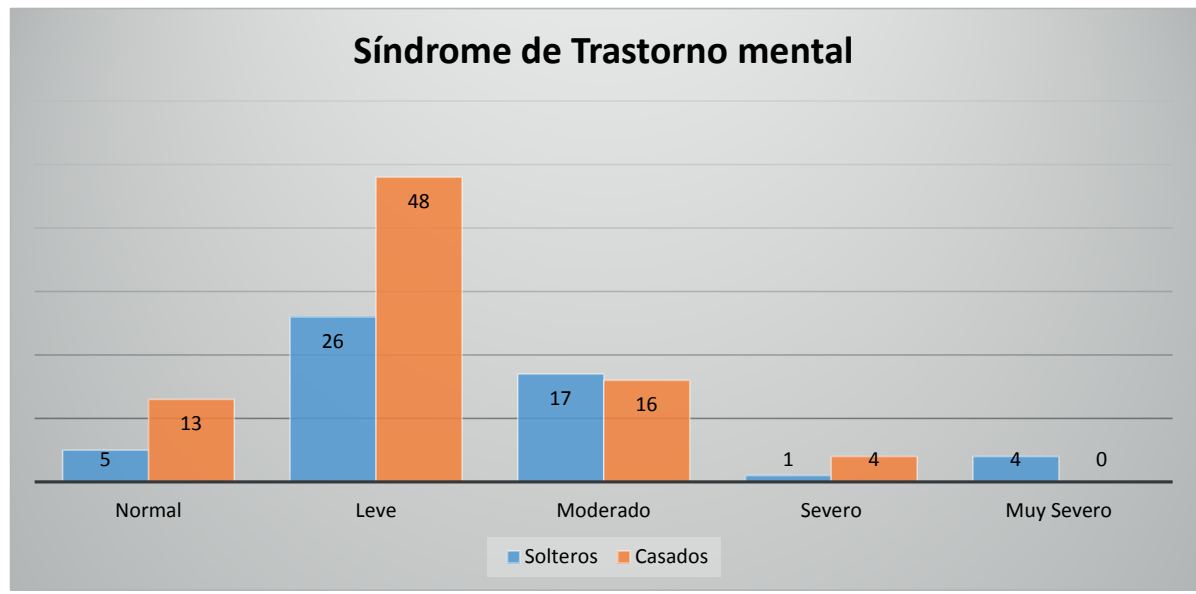

Fuente: Elaboración propia 
En la Gráfica 7 se presenta los niveles del Síndrome de Trastorno Mental y Género en la que en hombres se presenta de moderado a muy severo comparado con la mujeres esto implicaría que afecta la ansiedad, estrés y depresión se manifiesta en el género masculino.

\section{Gráfica 7 \\ Niveles de Síndrome de Trastorno Mental y Estado Civil}

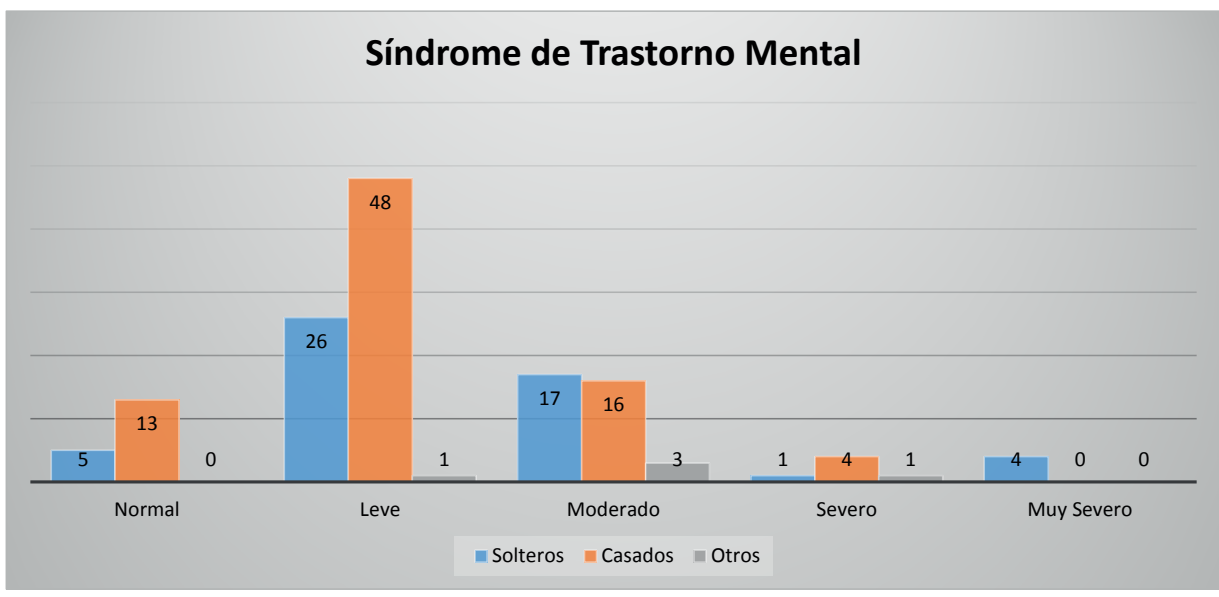

Fuente: Elaboración propia

En la Gráfica 7 se presenta los niveles del Síndrome de Trastorno Mental y Estado Civil en la que en personas solteras y casadas tiene un porcentaje nivel leve y moderado que en los otros casos de los encuestadas con el Dass 21 , en lo que se puede predecir que en las personas sin vínculo de esposa e hijos tienen ansiedad, depresión y estrés menor que los otros estados civiles.

La tabla 5, presenta los niveles del síndrome de Trastorno mental Dass 21 relacionado con los rangos de edad ante la presencia del Covid 19.

\section{Tabla 5}

\section{Niveles del Síndrome de Trastorno mental (Dass 21) por edad ante} el Covid 19 por edad

\begin{tabular}{cl}
\hline Rango de Edad (años) & Nivel del Síndrome de Trastorno mental (Dass 21) \\
\hline 13 a 22 & Normal $n=1$ \\
\hline 23 a 32 & $\begin{array}{l}\text { Normal } n=5, \text { Leve } n=35, \text { Moderado } n=25, \text { Severo } n= \\
1, \text { Muy Severo } n=3\end{array}$ \\
\hline
\end{tabular}


Cabezas-Heredia, Edmundo; Herrera-Chávez, Renato; Ricaurte-Ortiz, Paúl; Novillo Yahuarshungo, Carlos

Depresión, Ansiedad, estrés en estudiantes y docentes: Análisis a partir del Covid 19

\section{Cont... Tabla 5}

\begin{tabular}{cl}
\hline 33 a 42 & $\begin{array}{l}\text { Normal } n=10, \text { Leve } n=32, \text { Moderado } n=9, \text { Severo } n= \\
3, \text { Muy Severo } n=1\end{array}$ \\
\hline 34 a 52 & Normal $n=1$, Leve $n=6$, Moderado $n=2$, Severo $n=2$ \\
\hline 53 a 62 & Normal $n=1$, Leve $n=2$ \\
\hline
\end{tabular}

Fuente: Elaboración propia

La tabla 5 presenta los niveles del Síndrome de trastorno mental (Dass 21) de estudiantes y docentes por edad ante el Covid 19, donde se puede inferenciar que estos son moderado a severos muy pocos casos, se puede inferenciar que a pesar del confinamiento y presencia del virus Covid 19 los Trastornos de ansiedad, depresión y estrés son bajos, sin embargo al existir casos es necesario prevenir con atención especializad en el campo.

La tabla 6 presenta la prueba de normalidad de los datos de Kolmogorov - Smirnov para una muestra.

\section{Tabla 6}

Prueba de Normalidad

\begin{tabular}{|c|c|c|c|c|c|}
\hline \multicolumn{2}{|c|}{ Kolmogorov - Smirnov } & Depresión & Ansiedad & Estrés & $\begin{array}{c}\text { Síndrome } \\
\text { de Trastorno } \\
\text { Mental }\end{array}$ \\
\hline \multicolumn{2}{|l|}{$\mathrm{N}$} & 139 & 139 & 139 & 139 \\
\hline \multirow{2}{*}{$\begin{array}{l}\text { Parámetros norma- } \\
\text { les }^{a, b}\end{array}$} & Media &, 53 &, 40 & ,48 & ,47 \\
\hline & $\begin{array}{l}\text { Desviación } \\
\text { estándar }\end{array}$ &, 501 &, 536 &, 540 & ,489 \\
\hline \multicolumn{2}{|l|}{ Estadístico de prueba } & ,152 & ,230 & ,189 & 190 \\
\hline \multicolumn{2}{|c|}{ Sig. asintótica (bilateral) } &, $000^{c}$ &, $000^{c}$ &, $000^{c}$ &, $000^{c}$ \\
\hline \multicolumn{6}{|c|}{ a. La distribución de prueba es normal. } \\
\hline \multicolumn{6}{|c|}{ b. Se calcula a partir de datos. } \\
\hline
\end{tabular}

Fuente: Elaboración propia

Al ser una muestra de más de 50 encuestados se aplica Kolmogorov Smirnov para determinar la normalidad, donde todos los valores $p>0.05$ se concluye que existe normalidad.

Al aplicar Kolmogorov - Smirnov existe normalidad en los datos, al tratarse de dos variables cuantitativas 
con preguntas politómicas en escala de lickers, se determina el estadístico de Kruskal - Wallis con un $p$ valor significante de 0.05 que acepta la hipótesis de investigación y rechaza la nula.

La tabla 7 presenta la prueba de hipótesis de la investigación referente a:

\section{Tabla 7}

\section{Prueba de Kruskal - Wallis para muestras independientes}

Hipótesis nula
Prueba Wallis para muestras independientes
Prueba de Kruskal -

Sig.

Decisión

Rechazar la hipótesis nula

La distribución de Afrontamiento del COVID 19 (Agrupada) es la misma entre las categorías del Síndrome de Trastorno Mental
Nota: Se muestran significa8,000 ciones asintóticas. El nivel de significación es de 0.05

Fuente: Elaboración propia

Las variables cualitativas de estudio y preguntas politómicas determina la normalidad mediante Kruskal - Wallis con un $p$ valor significante de 0.05 donde se rechaza la hipótesis nula y se aprueba la de investigación.

La tabla 8 presenta el nivel de relacionamiento entre variables $y$ dimensiones de estudio.

Tabla 8

Nivel de relacionamiento Phi y V de Cramer

\begin{tabular}{lllr}
\hline & Medidas simétricas & \\
\hline & & Valor & $\begin{array}{c}\text { Significación } \\
\text { aproximada }\end{array}$ \\
\hline & Phi & 0.829 & 0.577 \\
\cline { 2 - 4 } Nominal por Nominal & V de Cramer & 0.479 & 0.577 \\
\cline { 2 - 4 } & $\begin{array}{l}\text { Coeficiente de } \\
\text { contingencia }\end{array}$ & 0.638 & 0.577 \\
\hline $\mathrm{N}$ de casos válidos & & & 139 \\
\hline
\end{tabular}

Fuente: Elaboración propia 
Cabezas-Heredia, Edmundo; Herrera-Chávez, Renato; Ricaurte-Ortiz, Paúl; Novillo Yahuarshungo, Carlos

Depresión, Ansiedad, estrés en estudiantes y docentes: Análisis a partir del Covid 19

Al realizar el análisis del índice de $\checkmark$ de Cramer que es de 0.577 se puede concluir que el efecto de relación entre variables y dimensiones es fuerte.

\section{Reflexiones Finales}

La presente investigación muestra que existe estrés, depresión y ansiedad en los estudiantes y docentes de posgrado de la Maestría en Prevención de Riesgos laborales de la UTPL con una capacidad de afrontamiento ante la presencia del Covid 19 media a bajo y con niveles escasos de alteraciones psicológicas que pueden ir cambiando en el tiempo. Esto implica que las personas se deben preparar psicológicamente mediante atención especializada, primeros auxilios, programas preventivos ante las posibles situaciones adversas que se tienen que convivir con el virus del Covid 19 y la pandemia en la actividad diaria de las personas. También implica prevenir y afrontar la crisis gestionando medidas socio sanitarias efectivas como el uso de mascarillas, distanciamiento, lavado de manos y sobre todo el apoyo psicológico (primeros auxilios psicológicos) para evitar el estrés postraumático.

Además, ante el confinamiento impuesto por el gobierno nacional y ante una posible situación de contagio, los niveles de estrés ansiedad y depresión aumentan en la población, de manera especial en personas vulnerables con posibles enfermedades como diabéticos, hipertensos, obesos, etc. Por ello, es importante desarrollar programas preventivos en ayuda psicológica que resguarden a estos grupos. Por otro lado, llama la atención que los niveles de afrontamiento sean bajos. Esto puede deberse a múltiples factores que se deberían investigar, es necesario crear programas gubernamentales, académicos de apoyo psicológico, con canales de información real, contextualizada que ayude a enfrentar la pandemia y no ahondar en generar pánico a las personas. También, es importante clasificar el contenido de la información que se recibe a través de las redes sociales y ayudarles a trabajar las competencias necesarias para poder filtrarlos.

El desconocimiento del Covid 19 a nivel de variables sociodemográficas $y$ afrontamiento tienen un porcentaje bajo de poco y ningún afrontamiento con pequeñas diferencia en género, nivel educativo y en la gente joven un mediano nivel de afrontamiento al Covid que se puede asumir debido a una mejor adaptación a las realidades problémicas.

Referente al Síndrome Traumático de estrés, ansiedad y depresión con las variables sociodemográficas género, edad, estado civil presenta porcentajes bajos con trastornos severos y muy severos derivados para primeros auxilios psicológicos y atención especializada de ser el caso.

La situación de confinamiento en la población de estudio es importante que sea atendida por los factores psicológicos de la ciudadanía. Se debe analizar los efectos que va a producir la crisis del COVID-19 en la salud de las personas. Por eso, se cree que este tipo de investigación puede ayudar a generar iniciativas de impacto social y sanitario de tratamiento para prevenir los efectos psicosociales de la pandemia para que la sociedad salga de la crisis.

Esta investigación ha tenido varias limitaciones por el confinamiento para la toma de datos que se ha solventado con herramientas informáticas como el Google forma para su aplicación, pero nos ha permitido ver la gravedad del 
riesgo y los efectos psicológicos sobre las personas la crisis que ha causado.

\section{Referencias Bibliográficas}

Apaza, P., Cynthia, M., Seminario, R.S., y Santa-Cruz, J.E. (2020). Factores psicosociales durante el confinamiento por el Covid-19 - Perú. Revista Venezolana de Gerencia, 25(90). http://www.redalyc. org/articulo.oa?id=29063559022.

Applegate, W.B., y Ouslander, J.G. (2020). COVID-19 presents high risk to older persons. J Am Geriatr Soc, 68(4), 681. https://doi.org/10.1111/ jgs. 16426

Antúnez Z, Vinet EV. (2012). Escalas de depresión, ansiedad estrés (DASS-21): validación de la versión abreviada en estudiantes universitarios chilenos. Terapia Psicológica, 30, 49-55.

APA. (2018). Ansiedad, depresión y estrés. $\quad$ http://www.apa.org/ centrodeapoyo/depresion.aspx.

Asmundson, G.J., y Taylor, S. (2020). Coronaphobia: fear and the 2019nCoV outbreak. J Anxiety Disord, 70, 102-96.

Bedoya-Lau, F.N., Matos, L. J., y Zelaya, E.C. (2014). Niveles de estrés académico, manifestaciones psicosomáticas y estrategias de afrontamiento en alumnos de la facultad de medicina de una universidad privada de Lima en el año 2012. Rev Neuropsiquiatr, 77(4), 262-270.

Beery, A.K., y Kaufer, D. (2015). Stress, social behavior, and resilience: insights from rodents. Neurobiol Stress, 1(1), 116-127. https://doi. org/10.1016/j.ynstr.2014.10.004

Brooks, S.K., Webster, R.K., Smith, L.E., Woodland. L., et al, (2020). The psychological impact of quarantine and how to reduce it: rapid review of the evidence. Lancet, 395, 912-20.

Camargo, M. (2020). Unos minutos para reflexionar sobre su 2020 (por su salud mental). EL COLOMBIANO S.A. \& CIA. S.C.A. https://www. elcolombiano.com/tendencias/ recomendaciones-de-salud-mentalpara-cerrar-el-2020-y-comenzar-el2021-ND14356694

Castellano, A. (2020). Efectos del COVID 19 en la colapsada economía venezolana del siglo XXI. Revista Venezolana de Gerencia, 25(91), 774 - 778. https://bit.ly/3sLvGL1

CEPAL. (2020). América Latina y el Caribe ante la pandemia del COVID 19. Santiago.

Chen Q, L. M. (2020). Mental health care for medical staff in China during the COVID-19 outbreak. Lancet Psychiatry, 7, e15-6.

Cova, F., Alvial, W., Aro, M., Bonifetti, A., y Hernández, M. (2007). Problemas de salud mental en estudiantes de la Universidad de Concepción. Ter Psicol , 25, 105-12.

Díaz, M. (2017). Determinar factores de riesgo psicosocial en docentes de bachillerato colegio Santa. ColombiaSoacha.

Dong, Y., Mo X, Hu Y, et al, (2020) Epidemiological characteristics of 2143 pediatric patients with 2019 coronavirus disease in China. American Academy of Pediatrics, https://doi.org/10.1542/peds.2020$\underline{0702}$

Ellis G, Gardner M, Tsiachristas A, Langhorne $\mathrm{P}$, Burke $\mathrm{O}$, Harwood $\mathrm{RH}$, Conroy SP, Kircher T, Somme D, Saltvedt I, Wald H, O'Neill D, Robinson D, Shepperd S (2017). Comprehensive geriatric 
Cabezas-Heredia, Edmundo; Herrera-Chávez, Renato; Ricaurte-Ortiz, Paúl;

Novillo Yahuarshungo, Carlos

Depresión, Ansiedad, estrés en estudiantes y docentes: Análisis a partir del Covid 19

assessment for older adults admitted to hospital. Cochrane Database of Systematic Reviews, 9. https://doi. org/10.1002/14651858.CD006211. pub3.

Idoiaga, N., De Montes, L.G., y Valencia, J. (2017). Understanding an ebola outbreak: social representations of emerging infectious diseases. $J$ Health Psychol, 22, 951-60.

Joffe, H. (2011). Public apprehension of emerging infectious diseases: are changes afoot? Public Underst Sci, 20, 446-60.

Liu S, Y. L.-T. (2020). Online mental health services in China during the COVID-19 outbreak.

Makamure, B., Mhaka, J., Makumbirofa, S., Mutetwa, R., Mupfumi, L., Mason, P., \& Metcalfe, J. Z. (2013). Microscopic-observation drugsusceptibility assay for the diagnosis of drug-resistant tuberculosis in Harare, Zimbabwe. PloS one, 8(2), e55872. https://doi.org/10.1371/ journal.pone.0055872 Lancet Psychiatry, 7:e17-8.

MSP. (2021, Febrero 19). Actualización de casos de coronavirus en Ecuador. Ministerio de Salud Pública del Ecuador. https://www.salud.gob. ec/actualizacion-de-casos-decoronavirus-en-ecuador/

Mukhtar, S. (2020). Psychological health during the coronavirus disease 2019 pandemic outbreak. International Journal of Social Psychiatry, 66(5). https://doi. org/10.1177/0020764020925835

OIT. (2020). Covid-19 y el mundo del trabajo: repercusiones y respuestas.

Regueiro, B., Rodríguez, S., Piñeiro, I., Estévez, I., Ferradás, M. Y Suárez, N. (2015) Diferencias en la percepción de la implicación parental en los deberes escolares en función del nivel de motivación de los estudiantes. European Journal of Investigation in Health, Psychology and Education, 5(3), 313-323.

Román, C. y Hernández. (2011). El estrés académico: una revisión crítica del concepto desde las ciencias de la educación. Revista Electrónica de Psicología Iztacala, 2 - 14

Sierra, J., Ortega, V., y Zubeidat, I. (2003). Ansiedad, angustia y estrés: tres conceptos a diferenciar. Revista Mal Estar e Subjetividade, 3(1). https://bit.ly/3ewdFuS

Shigemura J, Ursano RJ, Morganstein JC, Kurosawa M. (2020). Public responses to the novel 2019 coronavirus (2019-nCoV) in Japan: mental health consequences and target populations. Psychiatry Clin Neurosci, 74, 281-2.

Thakur, V., y Jain, A. (2020). COVID 2019-Suicides: Aglobal psychological pandemic. Brain Behavior and Immunity, 88. https://bit.ly/3tKPa3F

Van Bortel, T., Basnayake, A., Wurie, F., Jambai, M., Koroma, A. S., Muana, A. T., Hann, K., Eaton, J., Martin, S., \& Nellums, L. B. (2016). Psychosocial effects of an Ebola outbreak at individual, community and international levels. Bulletin of the World Health Organization, 94(3), 210-214. https://doi.org/10.2471/ BLT.15.158543

Wang, C., Pan, R., Wan, X., Tan, Y., Xu, L., y Ho, CS, et al, (2020). Immediate psychological responses and associated factors during the initial stage of the 2019 coronavirus disease (COVID-19) epidemic among the general population in China. Int. J, Environ Res Public Health, 17, 1729. 